\title{
PREOCUPACIONES Y CAMBIO SOCIAL DURANTE LA PANDEMIA DE CORONAVIRUS EN COLOMBIA
}

\author{
CONCERNS AND SOCIAL CHANGE DURING THE \\ CORONAVIRUS PANDEMIC IN COLOMBIA
}

\section{PREOCUPACÕES E MUDANCAS SOCIAIS DURANTE A PANDEMIA DE CORONAVÍRUS NA COLÔMBIA}

\author{
Oscar Javier Vergara Escobar ${ }^{1}$ \\ Néstor Camilo Castiblanco Alfonso ${ }^{2}$ \\ Juan David Montenegro Ramírez $z^{3}$ \\ Lina Rocío Corredor Parra ${ }^{4}$ \\ Anderson Reis de Sousa ${ }^{5}$ \\ Angie Vanessa Briñez Valderrama ${ }^{6}$
}

Como citar esse artículo: Escobar OJV, Alfonso NCC, Ramírez JDM, Parra LRC, Sousa AR, Valderrama AVB. Preocupaciones y cambio social durante la pandemia de coronavírus en Colombia. Rev baiana enferm. 2020; 34: e36953.

Objetivo: describir las preocupaciones y los cambios sociales de las personas durante la pandemia por coronavirus en Colombia. Método: estudio cuantitativo de tipo descriptivo-exploratorio, realizado con 352 personas en Colombia, por medio de un formulario estructurado de Google Forms en marzo de 2020. El análisis de datos se realizó por medio de estadística descriptiva. Resultados: el aislamiento social o físico ha generado un impacto en el estilo de vida de las personas en Colombia, lo que los ha llevado a tener preocupaciones que conducen a pensamientos rumiantes, asociados con factores generadores de estrés, relacionados con los problemas económicos, laborales y de salud de sus familiares. Conclusión: la pandemia de coronavirus ha causado preocupaciones, alteraciones y cambios consecuentes al estilo de vida de las personas en Colombia, situación que debe ser manejada interprofesional y holísticamente por profesionales de Enfermería.

Descriptores: Pandemia. Infecciones por Coronavírus. Cambio Social. Autopercepción

Objective: to describe the concerns and social changes of people during the coronavirus pandemic in Colombia. Method: quantitative descriptive-exploratory study, carried out with 352 people in Colombia, using a structured form on Google Forms in March 2020. The data analysis was done by descriptive statistics. Results: social or physical isolation has generated an impact on the lifestyle of people in Colombia, which has led them to have concerns that lead to ruminant thoughts, associated with stress generators, related to economic, labor and health problems of their families. Conclusion: the coronavirus pandemic has caused concerns, alterations and consequent changes to the lifestyle of people in Colombia, a situation that must be managed interprofessionally and bolistically by nursing professionals.

Descriptors: Pandemic. Coronavirus infections. Social change. Self-perception.

\footnotetext{
Enfermero. Maestría en Enfermería. Docente de la Escuela de Enfermería de la Fundación Universitaria Juan N Corpas, Bogotá, Cundinamarca, Colômbia. https:// orcid.org/0000-0003-3158-9017.

2 Enfermero. Maestría en Salud Mental Comunitaria. Docente de la Escuela de Enfermería de la Fundación Universitaria Juan N Corpas. https://orcid.org/0000-00024343-7865.

Enfermero. Maestría en Enfermería. Docente de la Fundación Universitaria Juan N Corpas. Bogotá, Cundinamarca- Colombia. https://orcid.org/0000-0002-9332-797। .

Enfermera. Maestría en Enfermería. Docente de la Escuela de Enfermería de la Fundación Universitaria Juan N Corpas. https://orcid.org/0000-0002-6565-086X.

Enfermero. Maestría en Enfermería. Docente de la Universidade Federal da Bahia. son.reis@hotmail.com. https://orcid.org/0000-000 I-8534-1960.

Enfermera. Maestría en Enfermería. Docente de la Escuela de Enfermería de la Fundación Universitaria Juan N Corpas. https://orcid.org/0000-000 I-9626-5457.
} 
Objetivo: descrever as preocupações e as mudanças sociais das pessoas durante a pandemia do coronavírus na Colômbia. Método: estudo quantitativo descritivo-explicativo, realizado com 352 pessoas na Colômbia, utilizando um formulário estruturado do Google Forms em março de 2020. A análise dos dados foi feita por meio de estatística descritiva. Resultados: o isolamento social ou físico gerou um impacto no estilo de vida das pessoas na Colômbia, o que as levou a ter preocupações que geram pensamentos ruminantes, associados a fatores de estresse relacionados a problemas econômicos, trabalhistas e de saúde de seus familiares. Conclusão: a pandemia de coronavirus causou preocupações, alterações e consequentes mudanças no estilo de vida das pessoas na Colômbia, uma situação que deve ser gerenciada interprofissional e holisticamente por profissionais de Enfermagem.

Descritores: Pandemia. Infecções por coronavírus. Mudança social. Auto-percepção.

\section{Introducción}

En diciembre de 2019, surgió un brote de enfermedades respiratorias en la provincia de Wuhan, en China, que llevó a los científicos chinos a identificar la aparición de un nuevo tipo de coronavirus que fue nombrado en febrero de 2020 como SARS-COV-2. Este virus causa la enfermedad conocida como Covid-19, que presenta un cuadro clínico que va desde infecciones asintomáticas hasta afecciones respiratorias graves, que llevan a las personas a requerir apoyo ventilatorio para el tratamiento de la insuficiencia respiratoria ${ }^{(1)}$.

Covid-19 se ha convertido en una pandemia debido a que es un agente altamente contagioso secundario a la vía de transmisión por gotas respiratorias contaminadas (aerosoles) entre personas que se encuentren a distancias cortas $(1,5 \mathrm{~m})$. El contacto prolongado es el factor de riesgo más alto, y el contagio de contactos casuales es menos probable. La mayoría de las infecciones se producen en pacientes sintomáticos, pero pueden ocurrir en pacientes asintomáticos e incluso en personas incubando la enfermedad $^{(2)}$. Este tipo de transmisión definitivamente hace que los seres humanos estén físicamente aislados, causando impactos sociales y personales, especialmente en los países más desiguales, como los indicadores demográficos más bajos, educación y salud ${ }^{(2)}$.

Una manera de minimizar los impactos causados por el contexto de la pandemia, los países, estados y municipios, es adoptar diferentes estrategias para la contención de la pandemia. Por lo general, se recomiendan las cuarentenas obligatorias o las barreras sociales y sanitarias como medidas de aislamiento para este tipo de transmisión: distancia entre pacientes, uso de habitación individual, uso de batas impermeables, guantes, gafas y máscaras quirúrgicas o tipo FFP2 para profesionales sanitarios, salvo en situaciones de especial riesgo ${ }^{(2-3)}$. Y si se trata de una decisión radical, esta medida fue la más eficaz para el control de la enfermedad, que en consecuencia afectó a la vida cotidiana del mundo $\mathrm{y}$, especialmente, de los microsistemas familiares, por lo que es esencial identificar y describir las preocupaciones, cambios sociales y necesidades de salud de las personas. Mientras tanto, estas medidas también están generando nuevos impactos, siendo merecedoras de investigación ${ }^{(4)}$.

En el escenario colombiano, la pandemia del nuevo Coronavirus ya ha alcanzado, hasta el 24 de mayo, 21.175 casos infectados, 5.016 personas recuperadas y 727 muertos, afectando a más de 24 Departamentos y/o Estados del país. Existe una gran preocupación por el avance de los nuevos casos diarios, así como con la evolución clínica de los casos activos y el número total de muertes por Coronavirus, teniendo en cuenta la posibilidad de impactos en el mantenimiento de la vida, el sistema de salud, la economía y otros pedidos esenciales a la condición humana ${ }^{(5)}$.

Teniendo en cuenta este panorama, se hace necesario justificar que los enfermeros deben dimensionar la posibilidad de la aparición de impactos pandémicos en las relaciones humanas en Colombia y su relación con la forma en que la población reacciona y enfrenta el contexto pandémico, es esencial identificar las preocupaciones y cambios sociales en un estilo de vida 
específico y único en cada territorio. Así, se observa que las personas y sus familias han estado en este aislamiento durante más de 90 días en los que se encuentra el comportamiento de la pandemia, pero al mismo tiempo se generan una serie de necesidades de esta primera necesidad, relacionadas con la forma de sobrevivir. Desde enfermería es una oportunidad para gestionar cuidados centrados en las necesidades de la persona y sus familias en las dimensiones de orden personal, familiar, social, espiritual, emocional, profesional y económico.

Por consiguiente, para hacerle frente a la pandemia, es necesario el aislamiento social en casa, lo que puede generar preocupaciones y cambios sociales importantes para afrontar las consecuencias económicas que trae el confinamiento, llevando a las personas y familias a presentar síntomas psicológicos y emocionales tales como ansiedad, depresión y preocupaciones financieras ${ }^{(6)}$.

Entendiendo la preocupación como una sensación que produce ansiedad, temor, angustia sobre algo que ha ocurrido, o que va a suceder, es una señal de alerta que advierte de un peligro inminente y permite a la persona tomar medidas para afrontar la amenaza $^{(7)}$. Y el cambio social definido como un cambio, alteración, modificación o evolución del comportamiento y la cultura humana, que con el tiempo, da lugar a consecuencias mensurables sobre los valores y normas de la sociedad ${ }^{(8)}$.

A partir de estos antecedentes, el grupo investigador plantea los siguientes vacíos del conocimiento, donde se desconoce en la actualidad las preocupaciones y los cambios sociales secundarios a la pandemia de Covid-19 en el contexto colombiano cuales son las dinámicas personales y de las familias colombianas frente al Covid-19, situaciones que permiten plantear estrategias de abordaje a las necesidades identificadas en estudios posteriores. Para investigar el fenómeno, este estudio se guio por la pregunta de investigación: ¿Cuáles son las preocupaciones y los cambios sociales de las personas durante la pandemia por coronavirus en Colombia? Para responder a esta pregunta, se plantea el objetivo de describir las preocupaciones y los cambios sociales de las personas durante la pandemia por coronavirus en Colombia.

\section{Metodología}

Este es un estudio cuantitativo descriptivo y exploratorio $^{(9-10)}$. Para el cálculo de la muestra se utilizó un muestro no probabilístico (conveniencia) el cual se seleccionó por ser una población accesible ya que están fácilmente disponibles ${ }^{(11)}$. Busca explicar las características del individuo, situaciones o grupos en particular, describiendo las preocupaciones y los cambios sociales de las personas que enfrentan el aislamiento social preventivo causado por la pandemia del Covid-19 en el contexto colombiano.

Para la preparación de este estudio, se consideraron los siguientes criterios de inclusión: tener más de 18 años, residir en el territorio nacional, tener un estado cognitivo conservado, tener acceso al servicio de internet y tener conocimientos básicos de gestión de las tecnologías de la información y la comunicación. Los criterios de exclusión fue personas que se identificaran con déficit cognitivo que le impide desarrollar la encuesta.

La realización de la encuesta digital $A d H o c$ se llevó a cabo en Google Forms, compuesta por ocho Ítems, una vez que la versión final fue revisada por un grupo de expertos e investigadores, su versión final quedó compuesta por 10 preguntas; que abordan las siguientes variables:

1. Caracterización de la población: edad, género, ocupación, ubicación geográfica dentro del territorio colombiano y afiliación al sistema de salud (subsidiado, contributivo, régimen especial y ninguno).

2. Descripción de las preocupaciones: relacionadas con lo que más preocupa a las personas por la situación actual de la pandemia, tales como: situación económica, académica y laboral, estado de salud, estado de salud de los familiares, ocupación del tiempo libre, estrés, falta de ejercicio físico y otros.

3. Descripción de los cambios sociales: durante el aislamiento social obligatorio por Covid-19. 
Relacionado con comer más de lo habitual; cambios en el ciclo de sueño; presencia de pesadillas; pasar más tiempo viendo la televisión; videojuego u otros.

Aunque se trata de un estudio de tipo descriptivo con una encuesta, se cumplió con los criterios éticos de la investigación, salvaguardando la confidencialidad, el anonimato, la fiabilidad y la veracidad de los datos generados.

Los datos se recopilaron entre el 22 de marzo y el 8 de abril de 2020, que fueron descargados de Excel y analizados con estadística descriptiva. Cabe destacar que el tema central de este estudio son las preocupaciones y los cambios sociales percibidos por las personas en cuarentena debido a la emergencia sanitaria mundial. No se centra en los procesos de intervención ni en los procedimientos biomédicos y, por lo tanto, la probabilidad de que los participantes sufran daños inmediatos o tardíos relacionados con la salud es mínima. Al comienzo de la investigación, se hace una breve introducción y se aclara que la persona que acepta completar está dando su consentimiento para que la información sea confidencial, utilizada con fines académicos y de investigación por un grupo de enfermeros.

Las consideraciones éticas para este estudio se basaron en la resolución 8430 de 1993, de la Republica Colombia, que establece normas científicas, técnicas y administrativas para la investigación sanitaria, clasificadas como de riesgo mínimo de acuerdo con el artículo 11, que describe la categoría como "estudios prospectivos que utilizan el registro de datos a través de procedimientos comunes consistentes en: pruebas de diagnóstico físico o psicológico o tratamientos de rutina" ${ }^{(12)}$. Como una forma de garantizar el rigor metodológico, adoptado los mejores criterios SQUIRE 2.0 a lo largo del proceso.

\section{Resultados}

La población participante fueron personas mayores de 18 años obteniendo 352 respuestas a través de la encuesta digital de Google Forms, donde se organizaron los resultados de acuerdo con las características de la población, las preocupaciones y los cambios sociales de las personas frente a la pandemia del Covid-19.

\section{Caracterización de la población}

El estudio incluyó 352 personas, 247 (70,2\%), mujeres y 104 (29,5\%) hombres. Los participantes forman parte de la población colombiana, con la participación de 22 de los 32 departamentos del país; el departamento de Cundinamarca tenía 270 (76,7\%), Boyacá y Casanare con 20 (5,7\%), Antioquia y Tolima con 7 (2\%); Meta con 4 (1,1\%), Bolívar, Cauca Córdoba, Huila, Magdalena, Norte de Santander, Santander y Valle del Cauca con 2 (0,6\%), Arauca, Caquetá, Cesar, Chocó, Guaviare, Nariño, Sucre y Quibdó con $1(0,3 \%)$.

El grupo de edad de 10 a 20 años fue de 16 personas (4,5\%), 21 a 30 años 108 (30,7\%), 31 a 40 años 119 (33,8\%), 41 a 50 años 71(20,2\%), 51 a 60 años $29(8,2 \%), 61$ a 70 años $5(1,4 \%)$ y más de 71 años 4 (1,1\%). La modalidad de afiliación al sistema de salud corresponde al régimen contributivo con 262 personas $(74,4 \%), 54$ subsidiadas (15,3\%), 24 en régimen especial $(6,8 \%)$ y no tenían seguridad social 12 (3,4\%). En cuanto a la situación laboral, 221 participantes (63\%) están empleados, 49 (14\%) son estudiantes; 33 (9\%) están desempleados; 28 (8\%) son independientes; 12 (3\%) son pensionistas y 9 (3\%) son amas de casa.

\section{Descripción de las preocupaciones}

Entre las principales preocupaciones que surgen en estas personas en relación con Covid-19 se identificó que 257 (73\%) informó de que la situación económica y la salud de los miembros de la familia son los aspectos que más les preocupan, la situación del trabajo y su propia salud, equivalentes al 40\% y en segundo lugar; $15 \%$ de los participantes identifican el estrés como un síntoma que pueden presentar durante esta situación específica; el 10\% informa que le preocupa la ocupación del tiempo libre 
y, por último, las personas que estudian están preocupadas por la situación académica que corresponde al $11 \%$.

Otros aspectos fueron relevantes, y los investigadores clasificaron las respuestas como: preocupaciones personales, relacionados con la salud mental, como ir a comprar medicamentos, la exposición a enfermedades, las preocupaciones y la falta de amor por Dios. Las preocupaciones del grupo surgen como, la salud de mi pareja, el suministro de alimentos, los usuarios crónicos, la situación de salud de la población, el aislamiento social, que hay personas que no se toman en serio el aislamiento, la falta de suministros en los hospitales, el sistema de salud defectuoso para proteger a los trabajadores. Ver Cuadro 1.

Cuadro 1 - Preocupación por Covid-19. Bogotá, Colombia - 2020. (N=352)

\begin{tabular}{|c|c|c|}
\hline Preocupaciones & $\mathbf{N}$ & $\%$ \\
\hline Situación económica & 257 & 73 \\
\hline Situación Laboral & 123 & 35 \\
\hline Situación académica & 40 & 11 \\
\hline Mi estado de salud & 140 & 40 \\
\hline Salud de familiares & 250 & 71 \\
\hline Ocupación de tiempo libre & 36 & 10 \\
\hline Estrés & 52 & 15 \\
\hline Ejercicio & 27 & 8 \\
\hline Otros & 14 & 4 \\
\hline
\end{tabular}

Fuente: Elaboración propia.

\section{Descripción de los cambios sociales:}

Los participantes, cuando se les preguntó si tuvieron cambios sociales durante el aislamiento físico debido a Covid-19, 51\% (179) responden que sus alteraciones son psicosociales, incluyendo cambios en la ansiedad, el estrés y el patrón de sueño; luego, están los cambios en la gestión de las actividades de ocio con 39\% (138), que ven más televisión, juegan videojuegos y utilizan las redes sociales y los medios de comunicación con más frecuencia; en tercer lugar, los cambios relacionados con el aumento del consumo de alimentos y/o la disminución en un 39\% (137). Y es sorprendente que sólo el 10\% (35) de las personas sean conscientes, en el momento de la respuesta, a afirmar que no presentan cambios inadecuados. Ver lo Cuadro 2.

Cuadro 2 - Cambios en el estilo de vida durante la pandemia. Bogotá, Colombia - 2020. (N=352)

\begin{tabular}{|l|c|c|}
\hline Cambio de estilos de vida & $\mathbf{N}$ & $\mathbf{\%}$ \\
\hline $\begin{array}{l}\text { Cambios en los hábitos nutricionales (aumento en el consumo de alimentos o } \\
\text { disminución). }\end{array}$ & 137 & 39 \\
\hline Cambios Psicosociales (ansiedad, estrés y alteración del patrón del sueño). & 179 & 51 \\
\hline $\begin{array}{l}\text { Cambios en el Ocio como televisión y video juegos (televisión, redes sociales y otros } \\
\text { medios de comunicación). }\end{array}$ & 138 & 39 \\
\hline Ninguno. & 35 & 10 \\
\hline
\end{tabular}

Fuente: Elaboración propia. 


\section{Discusión}

El nuevo Coronavirus ha sido catalogado por la Organización Mundial de la Salud como una emergencia en salud pública de importancia internacional (ESPII). En Colombia se confirmó el primer caso el 06 de marzo 2020. Se conoce que cualquier persona puede infectarse, independientemente de su edad o género, y cabe resaltar que se han registrado pocos casos en niños. La mortalidad han sido personas de edad avanzada que ya padecían una enfermedad crónica como diabetes, asma o hipertensión.

Teniendo en cuenta este contexto, refleja cuánto una enfermedad epidémica tiene un potencial para generar cambios abruptos e inesperados, que alteran en gran medida la vida cotidiana de las personas y su organización social. Afecta a la dinámica de las personas, al mundo del trabajo, a la producción tecnológica y genera nuevos hábitos, rutinas y comportamientos, que para muchos pueden ser perjudiciales, comprometiendo la situación sanitaria, especialmente la salud mental, debido al sufrimiento, la incertidumbre, el aislamiento social, la privación y otras lesiones ${ }^{(13)}$.

En cuanto a los hallazgos evidenciados en este estudio, se observa que la población más adherida a la investigación realizada por medios digitales es predominantemente joven, con acceso a internet utilizando una computadora $\mathrm{O}$ tableta y/o utilizando una red de telefonía móvil del tipo de teléfono inteligente; y que en la que las mujeres se movilizaron más para responder a esta investigación. Debido a este hallazgo, implica destacar una dimensión de clase y género asociada a la preocupación por los impactos generados por la pandemia, así como el acceso a los recursos tecnológicos disponibles para ello.

Por lo tanto, se refleja en el hecho de que las acciones basadas en el uso de recursos tecnológicos tendrán amplias posibilidades para el alcance de las poblaciones en el campo, residentes en ciudades menos pobladas, así como la población masculina, que aparece en un número menor con respecto a la participación en la investigación, así como ha demostrado un comportamiento más preocupante de los contextos pandémicos debido al hecho de que se adhieren a menos medidas de prevención y afrontamiento de enfermedades epidémicas, como ocurrió en los países con Brasil y Estados Unidos en la pandemia $\mathrm{H} 1 \mathrm{~N} 1^{(14)}$.

La población proviene de personas que están afiliadas al sistema de salud privado, están empleadas, a través de la identificación de este marcador económico, se cree que estas personas pueden enfrentar la pandemia de manera diferente a las que son una situación de mayor vulnerabilidad económica, especialmente el acceso a los servicios de salud, si es necesario. Además, los datos revelan la posibilidad de que estas personas sufran menos los impactos generados por la pandemia, especialmente en relación con el surgimiento de una crisis financiera.

Bajo el aspecto socioeconómico de las personas que están experimentando una pandemia con graves repercusiones como ha estado sucediendo con el contexto del nuevo Coronavirus, está la correlación entre el estado socioeconómico, la condición de salud y las respuestas físicas y psicoemocionales atribuidos a la contaminación y el desarrollo de Covid-19; resultados que son similares a los encontrados por Tull, quienes identificaron altos grados de depresión $\mathrm{y}$ ansiedad y preocupaciones socioeconómicas en personas confinadas en casa durante la cuarentena del Covid-19 en Estados Unidos ${ }^{(15)}$.

Se observa que en escenarios con personas de menor estatus socioeconómico se han presentado resultados desfavorables a la enfermedad. Además de esta dimensión, los marcadores están entrelazados con las esferas étnicas raciales, que identifican la mayor prevalencia de personas negras y trigueñas afectadas por la enfermedad, como países como Brasil y Estados Unidos. Como resultado, Colombia emitió un decreto, que estableció el aislamiento y el distanciamiento social a través de una cuarentena obligatoria de 20 días, que más tarde se extendió hasta el 11 de mayo. Después de determinar un aumento sostenido de nuevos casos de coronavirus, y dada la necesidad insatisfecha de acceso a los servicios básicos de salud, se 
reguló el Concepto Clínico Especializado (Consejo Clínico Virtual) aplicado como servicio de telemedicina ${ }^{(16)}$.

El impacto generado por el aislamiento social en la organización financiera de la población que sobrevive del comercio local, la producción manufacturada, la artesanía local, la agricultura familiar, y que, debido a este nuevo fenómeno, su subsistencia se ve comprometida. A nivel local, en Colombia el gobierno ha establecido estrategias para reducir los impactos. Como resultado de este problema, se teme que las desigualdades sociales y las inequidades intensificadas por la pandemia generen un aumento de la pobreza, el hambre y la mortalidad de la población debido a estas causas.

Como preocupaciones se han observado más tiempo en el hogar, conflictos familiares, vulnerabilidad femenina a la violencia, explotación y abuso infantil, abuso de alcohol y otras drogas, estigmatización, culpas e inequidades. Este preocupante escenario revela la necesidad desarrollo de acciones intersectoriales encaminadas a garantizar la protección social de las poblaciones, así como el avance de la cooperación técnica entre los países, de modo que no haya graves disminuciones en los indicadores de desarrollo humano, sostenibles, sociales y de derechos humanos, que estén en consonancia con la agenda internacional de los objetivos del milenio ${ }^{(17)}$.

En cuanto a las principales preocupaciones que surgieron de la población objetivo-alcanzada en este estudio, destacamos las dimensiones que afectan a la situación económica y familiar, que se superponen a las relacionadas con el mundo del trabajo y la propia situación sanitaria. Como factores que potencian los impactos, el estrés ha estado presente en ciertas situaciones específicas experimentadas desde la aparición de la pandemia y la determinación de medidas sanitarias de afrontamiento. Por otro lado, el exceso de tiempo libre ha llevado la preocupación de la población colombiana investigada en este estudio, ya que ésos se insertan activamente en el mercado laboral formal y que con cuarentena necesitan adaptar las actividades de uso del tiempo y desempeño laboral.
Dado que las personas que participaron en el estudio son educadas, el daño generado a la situación académica se convierte en un campo de gran preocupación para la población en Colombia. En resumen, parece que las preocupaciones se dirigieron a las dimensiones del orden personal, en las que se encuentran aspectos específicos de la autoprotección, la seguridad individual, así como en el ámbito espiritual y social, mediante el fortalecimiento de la creencia debido al temor a la falta de sensibilidad, la falta de amor y la falta de solidaridad colectiva hacia las personas, especialmente las que se encuentran en una situación más vulnerable a la devastación causada por la pandemia, que es durante el contexto pandémico, como en el período post pandémico. Además, cabe destacar también el hecho de que la población que participó en el estudio revela un sentimiento de preocupación relacionado con la capacidad de los hospitales para seguir siendo sostenibles para garantizar el mantenimiento de la atención sanitaria necesaria, así como el temor a la posibilidad de deficiencias en el sistema de salud en la protección de sus trabajadores y usuarios ${ }^{(17)}$.

Estudios previos afirman que ${ }^{(18)}$ individuos y comunidades, en mayor o menor medida relacionados con la epidemia Covid-19, la perciben como un evento estresante de vida y una situación amenazante que generó cambios psicológicos en la sociedad debido al confinamiento, el aislamiento físico, el cierre de escuelas y lugares. En el trabajo, esto genera un conjunto de síntomas importantes, como ansiedad, estrés, aburrimiento, miedo, ira y soledad; otros hallazgos relevantes son también para hipocondría, insomnio, miedos o incluso ataques generalizados de ansiedad y pánico ${ }^{(19)}$. Lo anterior invita al equipo a no omitir o ignorar el trauma causado por este evento, porque, durante muchas décadas, la salud mental de la población fue subestimada ${ }^{(20-21)}$.

Los datos identificaron la ocurrencia de cambios en el estilo de vida de la población colombiana después de la aparición de la pandemia, especialmente debido al aislamiento social, que hizo que las personas experimentaran un 
trastorno psicosocial, impulsando la enfermedad mental por la ocurrencia de sufrimiento, expresada en forma de trastornos de ansiedad, aumento del estrés, alteración del ciclo de vigilia del sueño y cambios inesperados, no programados $\mathrm{y}$, a veces, sin inspiración en las actividades de ocio que son utilizadas por estas personas como medidas para promover bien. Lo que se puede observar es que una vez aislados, las personas comenzaron a utilizar estrategias adaptativas al escenario experimentado, consumiendo, más de lo habitual, contenidos disponibles en la red de televisión, en los juegos de interacción ofrecidos por los videojuegos y el acceso a redes sociales como Instagram, Facebook, WhatsApp, que proporcionan la aproximación y expansión de las redes sociales comunicativas y promotores de afecto y vínculo ${ }^{(22)}$.

Asociado a estos cambios en la dinámica social de la vida cotidiana, hay un aumento en el número de comidas realizadas a lo largo del día, que es el resultado del confinamiento y las restricciones que limitan el acceso de la población a los espacios colectivos, que también afectan la inactividad física, el sedentarismo, el tabaquismo, el sobrepeso, la obesidad. En consecuencia, hay también el aumento de enfermedades crónicas no transmisibles, volviendo a un escenario que revela nuevos impactos que todos los países deben superar en la agenda sanitaria mundial.

El estrés es un factor predominante en este estudio, pero ahora este estrés ha dado lugar a comportamientos que alteran las relaciones personales, comunitarias y sociales que actúan sinérgicamente en la ocurrencia de violencia ${ }^{(23)}$, este estudio confirma que la crisis sanitaria, económica y social causada por la pandemia Covid-19 y sus necesarias medidas de afrontamiento pueden aumentar considerablemente el riesgo de violencia contra las mujeres ${ }^{(23-24)}$. Para muchas mujeres, las medidas de emergencia necesarias para combatir el Covid-19 aumentan las tareas domésticas y la atención a los niños enfermos, los ancianos y sus familias. Las restricciones de movimiento, las restricciones financieras y la inseguridad generalizada también fomentan a los agresores, dándoles mayor poder y control, situación reportada en los últimos días por las autoridades colombianas ${ }^{(23)}$.

Los cambios sociales durante el confinamiento resaltan las alteraciones psicosociales incluyendo cambios como la ansiedad, el estrés, el patrón del sueño, la gestión de actividades de ocio como permanecer más tiempo viendo televisión, uso de videojuegos y navegar por las redes sociales. Adicional a esto se ven cambios en la frecuencia del consumo de alimentos, llevándolos a estar más expuestos a un sin número de alteraciones que impactan directamente sobre la salud física y emocional de los individuos, familias y comunidades.

Los profesionales deben aportar a los planes de acción que reconozca el papel de la enfermería en el sistema de salud ${ }^{(25)} \mathrm{y}$, además, su papel en la salud de las comunidades y las familias es fundamental. En este escenario de la pandemia Covid-19, generar estrategias como la teleenfermería o la telesalud como desafíos para la atención son estrategias pertinentes para mostrar lo innovadores, creativos y pertinentes que pueden ser los profesionales de enfermería al cuidar a las personas y sus familias con conocimiento de las necesidades sentidas, liderazgo ${ }^{(26)}$ y pertinencia social, reto que desde las universidades debemos acoger con responsabilidad.

\section{Conclusiones}

El estudio describió las preocupaciones y los cambios sociales relacionadas con las necesidades de salud de las familias colombianas ante el surgimiento de la nueva pandemia de Coronavirus en el país. Los hallazgos revelan datos iniciales sobre el fenómeno de la salud, ya que su aprehensión ocurrió en los primeros días de la enfermedad epidémica que ocurre en el territorio colombiano, pero ya proporciona datos sustanciales relevantes para el conocimiento sobre la historia natural de la enfermedad en Colombia, como las dimensiones que generan preocupación por las personas y sus familias, así como los cambios sociales en la vida cotidiana y 
el estilo de vida. Esta situación debe ser manejada interprofesional y holísticamente.

El comportamiento social en el contexto de una pandemia confiere un conocimiento importante que debe emplearse a nivel de organizaciones de emergencia, contingencia, protección de las poblaciones afectadas, acciones del estado, con la formulación de políticas públicas que impacten en la salud física, social, emocional, la educación y la economía de un país a nivel geopolítico en el orden local, nacional e internacional.

La pandemia por la infección del coronavirus es percibida por los individuos y las comunidades como una situación estresante y amenazante para la vida, evidenciada en los cambios psicológicos, sociales y físicos que han sido, derivados del confinamiento, en donde se han presentado síntomas relevantes como la ansiedad, estrés, aburrimiento, miedo, ira y soledad. Es un reto para el equipo interprofesional intervenir de manera oportuna, integral y eficazmente los efectos que a corto, mediano y largo plazo que deja la pandemia.

Limitaciones del estudio permite ver que el número de participantes es bajo, siendo Colombia poblada por 50 644. 688 millones de habitantes. Además otra limitante es que no se logra percibir este fenómeno en la población rural y de quienes no cuentan con internet para responder la encuesta.

\section{Colaboraciones:}

1 - concepción del proyecto, análisis, interpretación de datos: Oscar Javier Vergara Escobar, Néstor Camilo Castiblanco Alfonso, Juan David Montenegro Ramírez, Lina Rocío Corredor Parra, Anderson Reis de Sousa e Angie Vanessa Briñez Valderrama;

2 - redacción del artículo y revisión crítica relevante del contenido intelectual: Oscar Javier Vergara Escobar, Néstor Camilo Castiblanco Alfonso, Juan David Montenegro Ramírez, Lina Rocío Corredor Parra, Anderson Reis de Sousa e Angie Vanessa Briñez Valderrama;
3 - aprobación final de la versión a ser publicada: Oscar Javier Vergara Escobar, Néstor Camilo Castiblanco Alfonso, Juan David Montenegro Ramírez, Lina Rocío Corredor Parra, Anderson Reis de Sousa e Angie Vanessa Briñez Valderrama.

\section{Referencia}

1. Rothan HA, Byrareddy SN. The epidemiology and pathogenesis of coronavirus disease (COVID-19) outbreak. J Autoimmun. 2020; 09:102433. DOI: https://doi.org/10.1016/j.jaut.2020.102433

2. Trilla A. Un mundo, una salud: la nueva epidemia de coronavirus COVID-19. Med Clin (Barc). 2020; 154(5):175-7. DOI: https://doi.org/10.1016/j. medcli.2020.02.002

3. Centro Europeo para la Prevención y el Control de Enfermedades (ECDC). Coronavirus novel [Internet]. 2020 [cited 2020 mar 12]. Disponible en: https://www.ecdc.europa. i/en/nine.

4. Bayham J, Fenichel EP. Impacto de los cierres de escuelas para COVID-19 en la fuerza laboral de salud de los Estados Unidos y la mortalidad neta: un estudio de modelización. Lancet Public Health. 2020; 2667(20):1-8. DOI: http://dx.doi. org/10.1016/S2468-2667(20)30082-7

5. Instituto Nacional de Salud. Ministerio de Salud y Protección Social de Colombia [Internet]. 2015 [cited 2020 Mar 12]. Disponible en: https://www. ins.gov.co/Noticias/Paginas.

6. Bernal-Ruiz D, Horta-Buitrago S. Cuidado de enfermería para la familia del paciente crítico desde la teoría de la comprensión facilitada. Enferm. univ. 2014; 11(4):154-163. DOI:10.1016/ S1665-7063(14)70928-5

7. Johnson M, Cuesta L, Tumas N. Emociones, preocupaciones y reflexiones frente a la pandemia del COVID-19 en Argentina, Ciencia y Saude Colectiva. 2020; 25(1): 2447-2546. DOI: https://doi. org/10.1590/1413-81232020256.1.10472020

8. Gallego R. Cambio social y estudios de agenda: Análisis crítico y algunas ideas para el estudio del caso Cubano. Comun. Soc [Internet]. 2016 [cited 2020 aug 12]; (25): 183-207. Available from: http:// www.scielo.org.mx/pdf/comso/n25/n25a8.pdf

9. Polit D, Hungler B. Investigación científica en ciencias de la salud. 6. ed. México: McGraw- Hill Interamericana; 2003. 
10. Burns N, Grove SK. Investigación en Enfermería. España: Elsevier; 2004.

11. Sampieri RH, Fernández-Collado C, Lucio PB. Metodología de la Investigación. 4. ed. Ciudad de México: McGraw-Hill; 2006.

12. Ministerio de Salud. Por la cual se establecen las normas científicas, técnicas y administrativos para la investigación en salud [Internet]. Colombia; 1993 [cited 2020 aug 08]. Available from: https://www. minsalud.gov.co/sites/rid/Lists/BibliotecaDigital/ RIDE/DE/DIJ/RESOLUCION-8430-DE-1993.pdf

13. Lucchini-Raies C, Márquez-Doren F, Unjidos NG, Véliz JC, Suazo DJ, Florechaes CC et al. Care during Breastfeeding: Perceptions of Mothers and Health Professionals. Invest. educ. enferm. 2019; 37(2): e09. DOI: http://dx.doi.org/10.17533/udea.iee. v37n2e09

14. Monteiro AKC, Pereira MCC, Santos JDM, Machado RS, Nogueira LT, Santos EMLRA. Efeito da intervenção educativa no pós-operatório de pessoas com estomias intestinais de eliminação: revisão sistemática. Enferm. glob. 2020; 19(57):648690. DOI: http://dx.doi.org/eglobal.19.1.368501.

15. Tull MT, Edmonds KA, Scamaldo KM, Richmond JR, Rose JP, Gratz KL. Psychological Outcomes Associated with Stay-at-Home Orders and the Perceived Impact of COVID-19 on Daily Life. Psychiatry Res. 2020; 289:113098. DOI: https://doi. org/10.1016/j.psychres.2020.113098

16. Márquez VJR. Teleconsulta en la pandemia por Coronavirus: desafíos para la telemedicina posCOVID-19 telemedicina en la era post-COVID-19. Rev. colomb. gastroenterol. 2020;35(supl 1):5-16. DOI: http://dx.doi.org/10.22516/25007440.543.

17. Sullivan R, Chalkidou K. Llamado urgente a un plan de salida: las consecuencias económicas y sociales de las respuestas a la pandemia de COVID-19 [Internet]. Center for Global Delevopment, 2020 [cited 2020 mar 12]. Available from: https://www. cgdev.org/blog/urgent-call-exit-plan-economicand-social-consequences-responses-covid-19pandemic.

18. Organización Mundial de la Salud. WHO Mental Health Gap Action Programme (mhGAP) [Internet]. 2020 [cited 2020 apr 20]. Available from: https:// www.who.int/mental_health/mhgap/en/

19. Duan L, Zhu G. Psychological interventions for people affected by the COVID-19 epidemic.
Lancet Psychiatry. 2020; 7(4):300-2. DOI: https:// doi.org/10.1016/S2215-0366(20)30073-0

20. Gardner P, Moaleff P. Psychological impact on SARS survivors: Critical review of the English language literature. Can Psychol. 2015; 56(1): 123135. DOI: http://dx.doi.org/10.1037/a0037973

21. Nobles J, Martin F, Dawson S, Moran P, J Savovic. The potential impact of COVID-19 on mental health outcomes and the implications for service solutions [Internet]. Natl Inst Heal Res. 2020 [cited 2020 aug 09]; 1:1-36. Available from: https://arc-w.nihr. ac.uk/Wordpress/wp-content/uploads/2020/04/ COVID-19-Rapid-ReviewCOVID-and-MentalHealth-FINAL.pdf

22. Sanz JIM. Uso de las TIC en orientación en los tiempos de COVID-19. Revista AOSMA [Internet]. 2020 [cited 2020 aug 08]; (2005):88-91. Available from: https://dialnet.unirioja.es/servlet/ articulo? codigo $=7381638$

23. Marques ES, Moraes CL, Hasselmann MH, Deslandes SF, Reichenheim ME. Violencia contra mujeres, niños y adolescentes en tiempos de pandemia por COVID-19: panorama, motivaciones y formas de afrontarla. Cad. Saúde Pública. 2020; 36(4): e00074420. DOI: http://dx.doi. org/10.1590/0102-311x00074420

24. Krug EG, Dahlberg LL, Mercy JA, Zwi AB. Informe mundial sobre la violencia y la salud. Rev. Inst. Med. trop. 2003; 45(3):130-130. DOI: https://doi. org/10.1590/S0036-46652003000300014.

25. Fuentes GP. Enfermería y COVID-19: reconocimiento de la profesión en tiempos de adversidad. Rev. colomb. enferm. [Internet]. 2020 [cited 2020 aug 08]; 19(1):1909-1621. Available from: https://dialnet.unirioja.es/servlet/ articulo? codigo $=7396850$

26. Menegaz JC, Fontes VMS. Executive coaching to the development of competences of nurse leaders. rev. min. enferm. 2020; 24: e-1281. DOI: http:// www.dx.doi.org/10.5935/1415-2762.20200010

Recibido: 25 de maio de 2020 Aprobado: 17 de julho de 2020 Publicado: 7 de outubro de 2020 
A Revista Baiana de Enfermagem utiliza a Licença Creative Commons - Atribuição-NãoComercial 4.0 Internacional. https://creativecommons.org/licenses/by-nc/4.0/

Este artigo é de acesso aberto distribuído sob os termos da Licença Creative Commons (CC BY-NC).

Esta licença permite que outros remixem, adaptem e criem a partir do seu trabalho para fins não comerciais. Embora os novos trabalhos tenham de lhe atribuir o devido crédito e não possam ser usados para fins comerciais, os usuários não têm de licenciar esses trabalhos derivados sob os mesmos termos. 